\title{
Exact solutions of unsteady Korteweg-de Vries and time regularized long wave equations
}

\author{
S M Rayhanul Islam', Kamruzzaman Khan ${ }^{1 *}$ and M Ali Akbar $^{2}$
}

\author{
* Correspondence: \\ k.khanru@gmail.com \\ ${ }^{1}$ Department of Mathematics, Pabna \\ University of Science \& Technology, \\ Pabna 6600, Bangladesh \\ Full list of author information is \\ available at the end of the article
}

\begin{abstract}
In this paper, we implement the $\exp (-\Phi(\xi))$-expansion method to construct the exact traveling wave solutions for nonlinear evolution equations (NLEEs). Here we consider two model equations, namely the Korteweg-de Vries ( $\mathrm{KdV}$ ) equation and the time regularized long wave (TRLW) equation. These equations play significant role in nonlinear sciences. We obtained four types of explicit function solutions, namely hyperbolic, trigonometric, exponential and rational function solutions of the variables in the considered equations. It has shown that the applied method is quite efficient and is practically well suited for the aforementioned problems and so for the other NLEEs those arise in mathematical physics and engineering fields. PACS numbers: 02.30.Jr, 02.70.Wz, 05.45.Yv, 94.05.Fq.

Keywords: The $\exp (-\Phi(\xi))$-expansion method; The TRLW equation; The KdV equation; NLEEs; Exact solutions
\end{abstract}

\section{Introduction}

Most of the real world problems are generally modeled by NLEEs. The study of exact traveling wave solutions for NLEEs play an important role in the study of nonlinear physical phenomena. Therefore, finding explicit solutions of physics equations is an important and interesting subject.

In this paper, we consider two NLEEs which have a great importance in mathematical physics. The first one is Korteweg de Vries equation, derived by Diederik Johannes Korteweg together with his PhD student Gustav de Vries, now well known as the KdV equation (Wazwaz 2009), having the simplest form

$$
u_{t}+u u_{x}+\delta u_{x x x}=0
$$

where $\delta$ is a nonzero constant. The term $u_{t}$ in this equation describes the time evolution of the wave propagating in one direction. Moreover, this equation incorporates two adversary effects: nonlinearity represented by $u u_{x}$ that accounts for steepening of the wave, and linear dispersion represented by $u_{x x x}$ that describes the spreading of the wave. Nonlinearity tends to localize the wave while dispersion spreads it out. The balance between these weak nonlinear steepening and dispersion effect formulate the solitons (Wazwaz 2009). The KdV equation is used to model the disturbance of the surface of shallow water in the presence of solitary waves. The $\mathrm{KdV}$ equation is a generic model for the study of weakly nonlinear long waves, incorporating leading order nonlinearity and dispersion (Wazwaz 2009; Marchant and Smyth 1996). Also, it 
describes surface waves of long wavelength and small amplitude on shallow water (Monro and Parkes 1999, 2000; Zakharov and Faddeev 1971).

And the second equation is the time regularized long wave (TRLW) equation proposed by Joseph and Egri (1977) and Jeffrey (1978), which is one of the alternative form of $\mathrm{KdV}$ equation, having the form

$$
u_{t}+u_{x}+\alpha u u_{x}+u_{x t t}=0
$$

where $u, t$ and $x$ denote the amplitude, time, and spatial coordinate respectively and $\alpha$ is a nonzero constant (Taghizade and Neirameh 2010; Taghizadeha et al. 2012). The TRLW equation shares many of the properties of the KdV equation. Bona and Chen (1999) have shown that the initial value problem for the TRLW equation is well-posed, and that for small-amplitude, long waves, solutions of (2) agree with solutions of (1). The Joseph-Egri (TRLW) equation plays a major role in the study of nonlinear waves since it describes the large number of important physical phenomena, such as shallow water waves and ion-acoustic plasma waves (Hereman 2011).

The exact solutions of NLEEs have been investigated by many authors who are interested in nonlinear physical phenomena which exist in all fields including mathematical physics and engineering fields, such as fluid mechanics, electrodynamics, chemical physics, chemical kinematics, plasma physics, elastic media, optical fibers, solid state physics, biology, and atmospheric and so on.

In recent years, many methods for obtaining explicit traveling and solitary wave solutions of NLEEs have been proposed, such as the extended tanh-method (Abdou 2007; Parkes and Duffy 1996; Yan 2001; Wang and Li 2005a), the F-function expansion method (Wang and Zhou 2003; Wang and Li 2005b), the exp-function expansion method (He and Wu 2006; Khan and Akbar 2014c), the generalized Riccati equation (Wang and Zhang 2007; Wang et al. 2007, Wang et al. 2005), the direct algebra method (Hereman et al. 1986), the complex hyperbolic function method (Zayed et al. 2008), the Modified Simple Equation Method (Khan and Akbar 2014b), the $\left(G^{\prime} / G\right)$-expansion Method (Taghizade and Neirameh 2010; Bekir 2008; Khan and Akbar 2014d; Islam et al. 2013; Wang et al. 2008; Zhang et al. 2008) and others. The objective of this paper is to use a new method which is called the $\exp (-\Phi(\xi))$-expansion method. This method is firstly proposed by which the traveling wave solutions of non-linear equations are obtained. The main idea of this method is that the traveling wave solutions of non-linear wave equations can be expressed as a polynomial in $\exp (-\Phi(\xi))$, where $\Phi(\xi)$ satisfies the ordinary differential equation $(\mathrm{ODE}) \Phi^{\prime}(\xi)=\exp (-\Phi(\xi))+\mu \exp (\Phi(\xi))+\lambda$, and $\xi=x+\omega t$. The degree of the polynomial can be determined by considering the homogeneous balance between the highest order derivatives and nonlinear terms appearing in the given nonlinear partial differential equation. It will be shown that more traveling wave solutions of many nonlinear evolution equations can be obtained by using the $\exp (-\Phi(\xi))$-expansion method.

The rest of the article has been prepared as follows: Description of the $\exp (-\Phi(\xi))$-expansion method; applications of $\exp (-\Phi(\xi))$-expansion method to find the exact solutions of unsteady Korteweg-de Vries and time regularized long wave equations, graphical representation, and conclusions. 


\section{Description of the exp(- $\Phi(\xi))$-expansion method}

In this section we will describe the algorithm of the $\exp (-\Phi(\xi))$-expansion method for finding traveling wave solutions of non linear evolution equations. Suppose that a non linear equation in two independent variables $x$ and $t$ is given by,

$$
P\left(u, u_{t}, u_{x}, u_{t t}, u_{x x}, u_{x t}, \ldots \ldots \ldots . .\right)=0, \quad x \in R, t>0
$$

where $u(\xi)=u(x, t)$ is an unknown function, $P$ is a polynomial of $u(x, t)$ and its partial derivatives in which the highest order derivatives and non linear terms are involved. In the following, we give the main steps of this method (Khan and Akbar 2014a).

Step 1. Combining the independent variables $x$ and $t$ into one variables $\xi=x \pm \omega t$, we suppose that

$$
u(x, t)=u(\xi) \quad \xi=x \pm \omega t,
$$

where $\omega \in R-\{0\}$ is the velocity of relative wave mode.

The traveling wave transformation Eq. (4) permits us to reduce Eq. (3) to the following ordinary differential equation (ODE),

$$
F\left(u, u^{\prime}, u^{\prime \prime}, \ldots \ldots \ldots\right)=0,
$$

where $F$ is a polynomial in $u(\xi)$ and its derivatives, whereas $u^{\prime}(\xi)=\frac{d u}{d \xi}, u^{\prime \prime}(\xi)=\frac{d^{2} u}{d \xi^{2}}$, and so on.

Step 2.We suppose that Eq. (5) has the formal solution

$$
u(\xi)=\sum_{i=0}^{n} A_{i}(\exp (-\Phi(\xi)))^{i}
$$

where $A_{i},(0 \leq i \leq n)$ are constants to be determined, such that $A_{n} \neq 0$, and $\Phi=\Phi(\xi)$ satisfies the following ODE

$$
\Phi^{\prime}(\xi)=\exp (-\Phi(\xi))+\mu \exp (\Phi(\xi))+\lambda .
$$

Eq. (7) gives the following solutions:

When $\lambda^{2}-4 \mu>0, \mu \neq 0$,

$$
\begin{aligned}
& \Phi(\xi)=\ln \left(\frac{-\sqrt{\left(\lambda^{2}-4 \mu\right)} \tanh \left(\frac{\sqrt{\left(\lambda^{2}-4 \mu\right)}}{2}(\xi+k)\right)-\lambda}{2 \mu}\right), \\
& \Phi(\xi)=\ln \left(\frac{-\sqrt{\left(\lambda^{2}-4 \mu\right)} \operatorname{coth}\left(\frac{\sqrt{\left(\lambda^{2}-4 \mu\right)}}{2}(\xi+k)\right)-\lambda}{2 \mu}\right),
\end{aligned}
$$

When $\lambda^{2}-4 \mu<0, \mu \neq 0$,

$$
\Phi(\xi)=\ln \left(\frac{\sqrt{\left(4 \mu-\lambda^{2}\right)} \tan \left(\frac{\sqrt{\left(4 \mu-\lambda^{2}\right)}}{2}(\xi+k)\right)-\lambda}{2 \mu}\right)
$$




$$
\Phi(\xi)=\ln \left(\frac{-\sqrt{\left(4 \mu-\lambda^{2}\right)} \cot \left(\frac{\sqrt{\left(4 \mu-\lambda^{2}\right)}}{2}(\xi+k)\right)-\lambda}{2 \mu}\right),
$$

When $\lambda^{2}-4 \mu>0, \mu=0, \lambda \neq 0$,

$$
\Phi(\xi)=-\ln \left(\frac{\lambda}{\exp (\lambda(\xi+k))-1}\right),
$$

When $\lambda^{2}-4 \mu=0, \mu \neq 0, \lambda \neq 0$,

$$
\Phi(\xi)=\ln \left(-\frac{2(\lambda(\xi+k)+2)}{\lambda^{2}(\xi+k)}\right),
$$

When $\lambda^{2}-4 \mu=0, \mu=0, \lambda=0$,

$$
\Phi(\xi)=\ln (\xi+k),
$$

where $k$ is an arbitrary constant and $A_{n}, \omega, \lambda, \mu$ are constants to be determined later, $A_{n} \neq 0$, the positive integer $n$ can be determined by considering the homogeneous balance between the highest order derivatives and the nonlinear terms appearing in Eq. (5).

Step 3. We substitute Eq. (6) into Eq. (5) and then we account the function exp $(-\Phi(\xi))$. As a result of this substitution, we get a polynomial of $\exp (-\Phi(\xi))$. We equate all the coefficients of same power of $\exp (-\Phi(\xi))$ to zero. This procedure yields a system of algebraic equations whichever can be solved to find $A_{n}, \omega, \lambda, \mu$. Substituting the values of $A_{n}, \omega, \lambda, \mu$ into Eq. (6) along with general solutions of Eq. (7) completes the determination of the solution of Eq. (3).

\section{Applications}

The KdV equation

In this subsection we will apply $\exp (-\Phi(\xi))$-expansion method to construct analytical solutions of the KdV equation of the form (1).

The traveling wave transformation equation

$$
u=u(x, t), \xi=x-\omega t, u=u(\xi), u(x, t)=u(\xi),
$$

transforms Eq. (1) into the following ODE,

$$
-\omega u^{\prime}+u u^{\prime}+\delta u^{\prime \prime \prime}=0 .
$$

Integrating Eq. (16) with respect to $\xi$ once, yields

$$
C-\omega u+\frac{u^{2}}{2}+\delta u^{\prime \prime}=0,
$$

where $C$ is integrating constant that can be determine later.

Now taking the homogeneous balance between the highest order derivative $u$ " and the nonlinear term $u^{2}$ in Eq. (17), yields

$$
u(\xi)=A_{0}+A_{1}(\exp (-\Phi(\xi)))+A_{2}(\exp (-\Phi(\xi)))^{2},
$$

where $A_{0}, A_{1}$ and $A_{2}$ are constants to be determined such that $A_{2} \neq 0$, while $\lambda$ and $\mu$ are arbitrary constants. 
Substituting $u, u^{2}, u^{\prime \prime}$ into Eq. (17) and then equating the coefficients of $\exp (-\Phi(\xi))$ to zero, we obtain

$$
\begin{aligned}
& C-\omega A_{0}+\frac{1}{2} A_{0}^{2}+2 \delta A_{2} \mu^{2}+\delta A_{1} \mu \lambda=0 . \\
& \delta A_{1} \lambda^{2}+A_{0} A_{1}-\omega A_{1}+2 \delta A_{1} \mu+6 \delta A_{2} \mu \lambda=0 . \\
& -\omega A_{2}+\frac{1}{2} A_{1}^{2}+A_{0} A_{2}+3 \delta A_{1} \lambda+8 \delta A_{2} \mu+4 \delta A_{2} \lambda^{2}=0 . \\
& 10 \delta A_{2} \lambda+2 \delta A_{1}+A_{1} A_{2}=0 . \\
& \frac{1}{2} A_{2}^{2}+6 \delta A_{2}=0 .
\end{aligned}
$$

Solving the above five algebraic equations, yields

$$
\begin{aligned}
& C=\frac{1}{2} \omega^{2}-\frac{1}{2} \delta^{2} \lambda^{4}+4 \delta^{2} \lambda^{2} \mu-8 \delta^{2} \mu^{2}, A_{0}=\omega-\delta \lambda^{2}-8 \delta \mu \\
& A_{1}=-12 \delta \lambda, A_{2}=-12 \delta
\end{aligned}
$$

where $\lambda$ and $\mu$ are arbitrary constants.

Substituting the values of $C, A_{0}, A_{1}$ and $A_{2}$ into Eq. (18), yields

$$
u(\xi)=\omega-\delta \lambda^{2}-8 \delta \mu-12 \delta \lambda \exp (-\Phi(\xi))-12 \delta \exp (-2 \Phi(\xi))
$$

where $\xi=x-\omega t$

Now applying Eq. (8) to Eq. (14) into Eq. (24) respectively, we obtain the following seven traveling wave solutions of the KdV equation.

When $\lambda^{2}-4 \mu>0, \mu \neq 0$,

$$
\begin{aligned}
u_{1}(\xi)=\omega & -\delta \lambda^{2}-8 \delta \mu+\frac{24 \delta \lambda \mu}{\sqrt{\lambda^{2}-4 \mu} \tanh \left(\frac{1}{2} \sqrt{\lambda^{2}-4 \mu}(x-\omega t+k)\right)+\lambda} \\
& -\frac{48 \delta \mu^{2}}{\left(\sqrt{\lambda^{2}-4 \mu} \tanh \left(\frac{1}{2} \sqrt{\lambda^{2}-4 \mu}(x-\omega t+k)\right)+\lambda\right)^{2}} \\
u_{2}(\xi)=\omega & -\delta \lambda^{2}-8 \delta \mu+\frac{24 \delta \lambda \mu}{\sqrt{\lambda^{2}-4 \mu} \operatorname{coth}\left(\frac{1}{2} \sqrt{\lambda^{2}-4 \mu}(x-\omega t+k)\right)+\lambda} \\
& -\frac{48 \delta \mu^{2}}{\left(\sqrt{\lambda^{2}-4 \mu} \operatorname{coth}\left(\frac{1}{2} \sqrt{\lambda^{2}-4 \mu}(x-\omega t+k)\right)+\lambda\right)^{2}}
\end{aligned}
$$

When $\lambda^{2}-4 \mu<0, \mu \neq 0$,

$$
\begin{aligned}
& u_{3}(\xi)=\omega-\delta \lambda^{2}-8 \delta \mu-\frac{24 \delta \lambda \mu}{\left(\sqrt{4 \mu-\lambda^{2}} \tan \left(\frac{1}{2} \sqrt{4 \mu-\lambda^{2}}(x-\omega t+k)\right)-\lambda\right)} \\
& -\frac{48 \delta \mu^{2}}{\left(\sqrt{4 \mu-\lambda^{2}} \tan \left(\frac{1}{2} \sqrt{4 \mu-\lambda^{2}}(x-\omega t+k)\right)-\lambda\right)^{2}}
\end{aligned}
$$




$$
\begin{gathered}
u_{4}(\xi)=\omega-\delta \lambda^{2}-8 \delta \mu-\frac{24 \delta \lambda \mu}{\left(\sqrt{4 \mu-\lambda^{2}} \cot \left(\frac{1}{2} \sqrt{4 \mu-\lambda^{2}}(x-\omega t+k)\right)-\lambda\right)} . \\
-\frac{48 \delta \mu^{2}}{\left(\sqrt{4 \mu-\lambda^{2}} \cot \left(\frac{1}{2} \sqrt{4 \mu-\lambda^{2}}(x-\omega t+k)\right)-\lambda\right)^{2}} .
\end{gathered}
$$

When $\lambda^{2}-4 \mu>0, \mu=0, \lambda \neq 0$

$$
u_{5}(\xi)=\omega-\delta \lambda^{2}-\frac{12 \delta \lambda^{2}}{(\exp (\lambda(x-\omega t+k))-1)}-\frac{12 \delta \lambda^{2}}{(\exp (\lambda(x+\omega t+k))-1)^{2}} .
$$

When $\lambda^{2}-4 \mu=0, \mu \neq 0, \lambda \neq 0$,

$$
u_{6}(\xi)=\omega-3 \delta \lambda^{2}+\frac{6 \delta \lambda^{3}(x-\omega t+k)}{(\lambda(x-\omega t+k)+2)}-\frac{3 \delta \lambda^{4}(x-\omega t+k)^{2}}{(\lambda(x-\omega t+k)+2)^{2}} .
$$

When $\lambda^{2}-4 \mu=0, \mu=0, \lambda=0$,

$$
u_{7}(\xi)=\omega-\frac{12 \delta}{(x-\omega t+k)^{2}} \text {. }
$$

\section{The TRLW equation}

In this subsection, we will apply the $\exp (-\Phi(\xi))$-expansion method to find the exact solutions and then the solitary wave solutions of the TRLW equation of the form (2).

The traveling wave transformation equation is

$$
u=u(x, t), \xi=x+\omega t, u=u(\xi), u(x, t)=u(\xi),
$$

Eq. (25) transforms Eq. (2) into the following ODE,

$$
(1+\omega) u^{\prime}+\alpha u u^{\prime}+\omega^{2} u^{\prime \prime \prime}=0 .
$$

Integrating with respect to $\xi$, Eq. (26) yields

$$
C+(1+\omega) u+\frac{\alpha}{2} u^{2}+\omega^{2} u^{\prime \prime}=0
$$

where $C$ is the constant of integration.

Now balancing the highest order derivative $u$ " and non linear term $u^{2}$, we obtain $n=2$.

Hence for $n=2$, Eq. (6) yields

$$
u(\xi)=A_{0}+A_{1}(\exp (-\phi(\xi)))+A_{2}(\exp (-\phi(\xi)))^{2},
$$

where $A_{0}, A_{1}$ and $A_{2}$ are constants to be determined such that $A_{2} \neq 0$, while $\lambda$ and $\mu$ are arbitrary constants.

Substituting $u, u^{2}, u^{\prime \prime}$ into Eq. (27) and then equating the coefficients of $\exp (-\Phi(\xi))$ to zero, we obtain

$$
\begin{aligned}
& C-\omega A_{0}+\frac{1}{2} \alpha A_{0}^{2}+2 \omega^{2} A_{2} \mu^{2}+A_{0}+\omega^{2} A_{1} \mu \lambda=0 . \\
& \omega^{2} A_{1} \lambda^{2}+A_{1}+\alpha A_{0} A_{1}+\omega A_{1}+2 \omega^{2} A_{1} \mu+6 \omega^{2} A_{2} \mu \lambda=0 .
\end{aligned}
$$




$$
\begin{aligned}
& \omega A_{2}+3 \omega^{2} A_{1} \lambda+\frac{1}{2} \alpha A_{1}^{2}+\alpha A_{0} A_{2}+A_{2}+8 \omega^{2} A_{2} \mu+4 \omega^{2} A_{2} \lambda^{2}=0 . \\
& 10 \omega^{2} A_{2} \lambda+2 \omega^{2} A_{1}+\alpha A_{1} A_{2}=0 . \\
& \frac{1}{2} \alpha A_{2}^{2}+6 \omega^{2} A_{2}=0 .
\end{aligned}
$$

Solving the above five equations, yields

$$
\begin{aligned}
& C=-\frac{1}{2} \frac{-2 \omega-\omega^{2}-1+16 \omega^{4} \mu^{2}-8 \omega^{4} \lambda^{2} \mu+\omega^{4} \lambda^{4}}{\alpha}, A_{0}=-\frac{1+8 \omega^{2} \mu+\omega+\omega^{2} \lambda^{2}}{\alpha} \\
& A_{1}=-\frac{12 \omega^{2} \lambda}{\alpha}, A_{2}=-\frac{12 \omega^{2}}{\alpha}
\end{aligned}
$$

where $\lambda$ and $\mu$ are arbitrary constants.

Now substituting the values of $C, A_{0}, A_{1}$ and $A_{2}$ into Eq. (28) yields

$$
u(\xi)=-\left(\frac{1+8 \omega^{2} \mu+\omega+\omega^{2} \lambda^{2}}{\alpha}+\frac{12 \omega^{2} \lambda}{\alpha} \exp (-\Phi(\xi))+\frac{12 \omega^{2}}{\alpha} \exp (-2 \Phi(\xi))\right)
$$

where $\xi=x+\omega t$.

Substituting Eq. (8)-Eq. (14) into Eq. (34) respectively, we obtain the following seven traveling wave solutions of the TRLW equation.

When $\lambda^{2}-4 \mu>0, \mu \neq 0$,

$$
\begin{aligned}
u_{1}(\xi)=- & \frac{1+8 \omega^{2} \mu+\omega+\omega^{2} \lambda^{2}}{\alpha}+\frac{24 \omega^{2} \lambda \mu}{\alpha\left(\sqrt{\lambda^{2}-4 \mu} \tanh \left(\frac{1}{2} \sqrt{\lambda^{2}-4 \mu}(x+\omega t+k)\right)+\lambda\right)} . \\
& -\frac{48 \omega^{2} \mu^{2}}{\alpha\left(\sqrt{\lambda^{2}-4 \mu} \tanh \left(\frac{1}{2} \sqrt{\lambda^{2}-4 \mu}(x+\omega t+k)\right)+\lambda\right)^{2}} \\
u_{2}(\xi)=- & \frac{1+8 \omega^{2} \mu+\omega+\omega^{2} \lambda^{2}}{\alpha}+\frac{24 \omega^{2} \lambda \mu}{\alpha\left(\sqrt{\lambda^{2}-4 \mu} \operatorname{coth}\left(\frac{1}{2} \sqrt{\lambda^{2}-4 \mu}(x+\omega t+k)\right)+\lambda\right)} . \\
& -\frac{48 \omega^{2} \mu^{2}}{\alpha\left(\sqrt{\lambda^{2}-4 \mu} \operatorname{coth}\left(\frac{1}{2} \sqrt{\lambda^{2}-4 \mu}(x+\omega t+k)\right)+\lambda\right)^{2}}
\end{aligned}
$$

When $\lambda^{2}-4 \mu<0, \mu \neq 0$,

$$
\begin{aligned}
u_{3}(\xi)= & -\frac{1+8 \omega^{2} \mu+\omega+\omega^{2} \lambda^{2}}{\alpha}-\frac{24 \omega^{2} \lambda \mu}{\alpha\left(\sqrt{4 \mu-\lambda^{2}} \tan \left(\frac{1}{2} \sqrt{4 \mu-\lambda^{2}}(x+\omega t+k)\right)-\lambda\right)} . \\
& -\frac{48 \omega^{2} \mu^{2}}{\alpha\left(\sqrt{4 \mu-\lambda^{2}} \tan \left(\frac{1}{2} \sqrt{4 \mu-\lambda^{2}}(x+\omega t+k)\right)-\lambda\right)^{2}}
\end{aligned}
$$



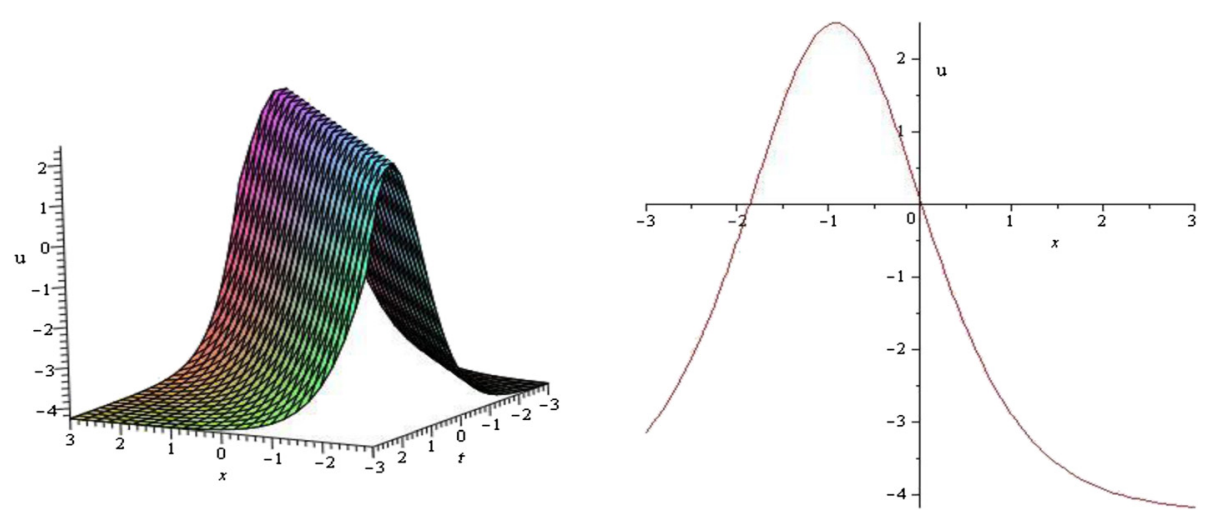

Figure 1 Bell shaped Soliton profile of $\mathrm{KdV}$ equation for $\lambda=3, \mu=1, k=0, a=2, \delta=1$ and wave speed $\boldsymbol{\omega}=\mathbf{1}$ within the interval $-\mathbf{3} \leq x, \boldsymbol{t} \leq \mathbf{3}$. (only shows the shape of $u_{1}(\zeta)$ ), The left figure shows the $3 \mathrm{D}$ plot and the right figure shows the $2 \mathrm{D}$ plot for $t=0$.

$$
\begin{aligned}
u_{4}(\xi)=- & \frac{1+8 \omega^{2} \mu+\omega+\omega^{2} \lambda^{2}}{\alpha}-\frac{24 \omega^{2} \lambda \mu}{\alpha\left(\sqrt{4 \mu-\lambda^{2}} \cot \left(\frac{1}{2} \sqrt{4 \mu-\lambda^{2}}(x+\omega t+k)\right)-\lambda\right)} \\
& -\frac{48 \omega^{2} \mu^{2}}{\alpha\left(\sqrt{4 \mu-\lambda^{2}} \cot \left(\frac{1}{2} \sqrt{4 \mu-\lambda^{2}}(x+\omega t+k)\right)-\lambda\right)^{2}}
\end{aligned}
$$

When $\lambda^{2}-4 \mu>0, \mu=0, \lambda \neq 0$,

$$
u_{5}(\xi)=-\frac{1+\omega+\omega^{2} \lambda^{2}}{\alpha}-\frac{12 \omega^{2} \lambda^{2}}{\alpha(\exp (\lambda(x+\omega t+k))-1)}-\frac{12 \omega^{2} \lambda^{2}}{\alpha(\exp (\lambda(x+\omega t+k))-1)^{2}}
$$

When $\lambda^{2}-4 \mu=0, \mu \neq 0, \lambda \neq 0$,

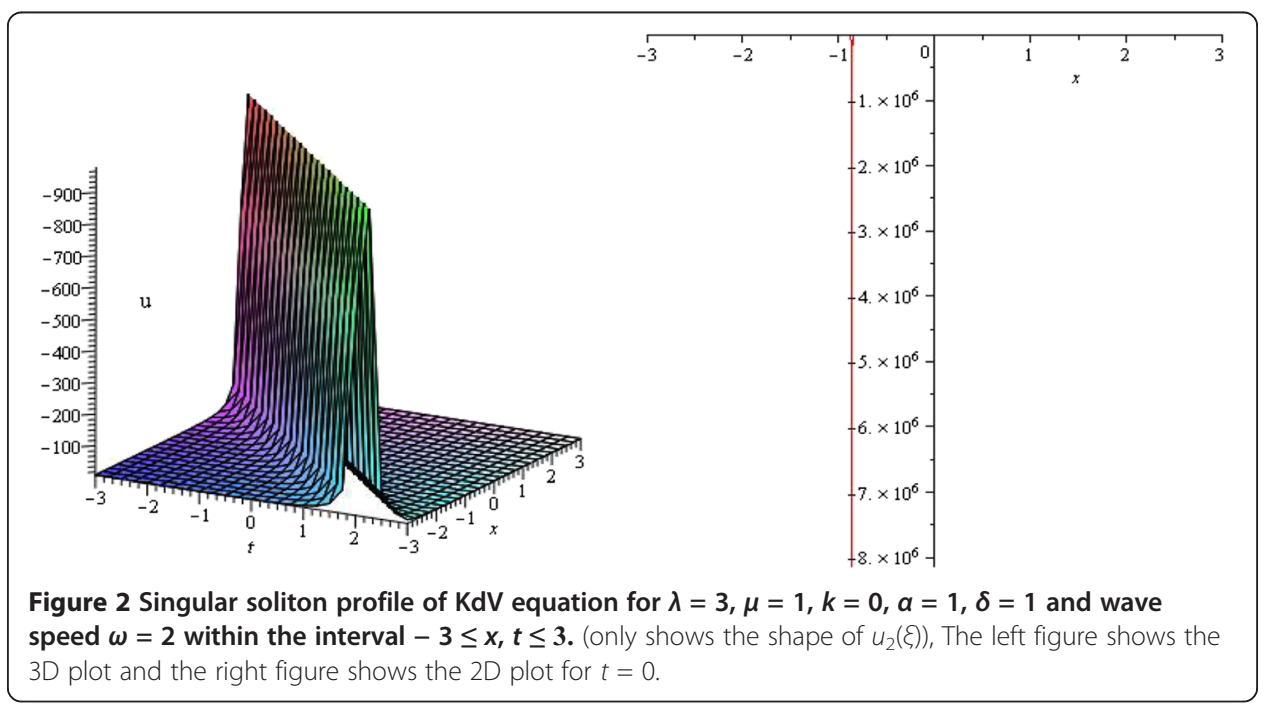



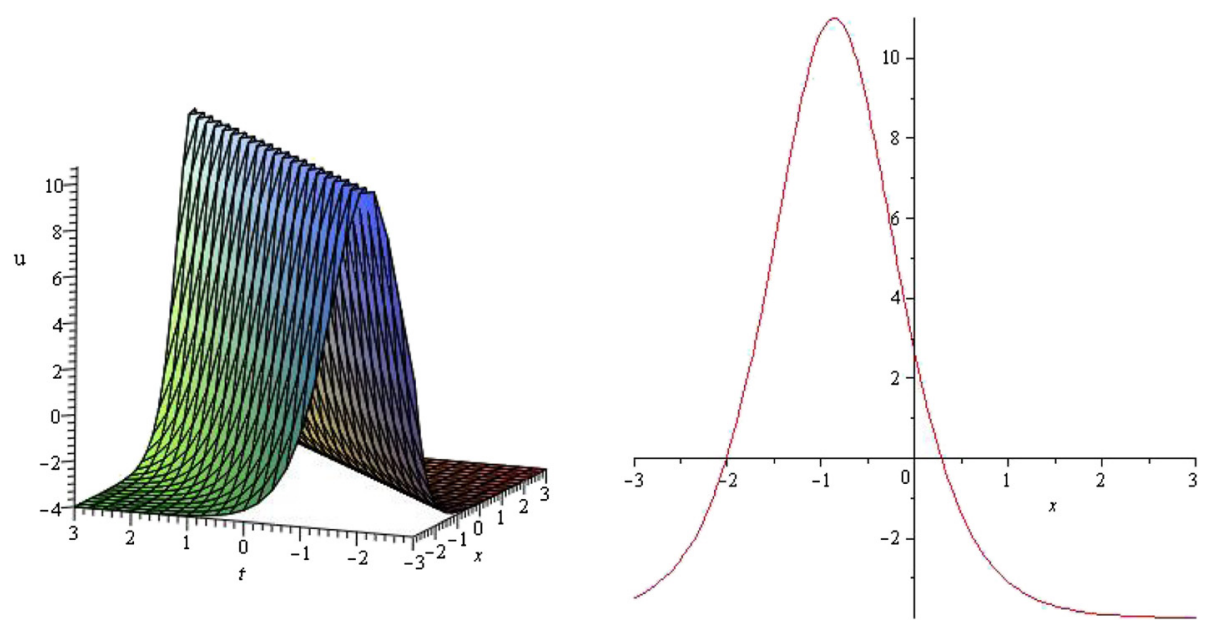

Figure 3 Bell shaped Soliton profile of TRLW equation for $\lambda=3, \mu=1, k=0, a=1$ and wave speed $\boldsymbol{\omega}=\mathbf{1}$ within the interval $-\mathbf{3} \leq \boldsymbol{x}, \boldsymbol{t} \leq \mathbf{3}$. (only shows the shape of $u_{1}(\xi)$ ), The left figure shows the 3D plot and the right figure shows the $2 \mathrm{D}$ plot for $t=0$.

$$
u_{6}(\xi)=-\frac{1+\omega+3 \omega^{2} \lambda^{2}}{\alpha}+\frac{6 \omega^{2} \lambda^{3}(x+\omega t+k)}{\alpha(\lambda(x+\omega t+k)+2)}-\frac{3 \omega^{2} \lambda^{4}(x+\omega t+k)^{2}}{\alpha(\lambda(x+\omega t+k)+2)^{2}} .
$$

When $\lambda^{2}-4 \mu=0, \mu=0, \lambda=0$,

$$
u_{7}(\xi)=-\frac{1+\omega}{\alpha}-\frac{12 \omega^{2}}{\alpha(x+\omega t+k)^{2}} .
$$

\section{Graphical representation of some obtained solutions}

Using mathematical software Maple, 2D and 3D plots of some obtained solutions have been shown in Figures 1, 2, 3 and 4 to visualize the underlying mechanism of the original equations.

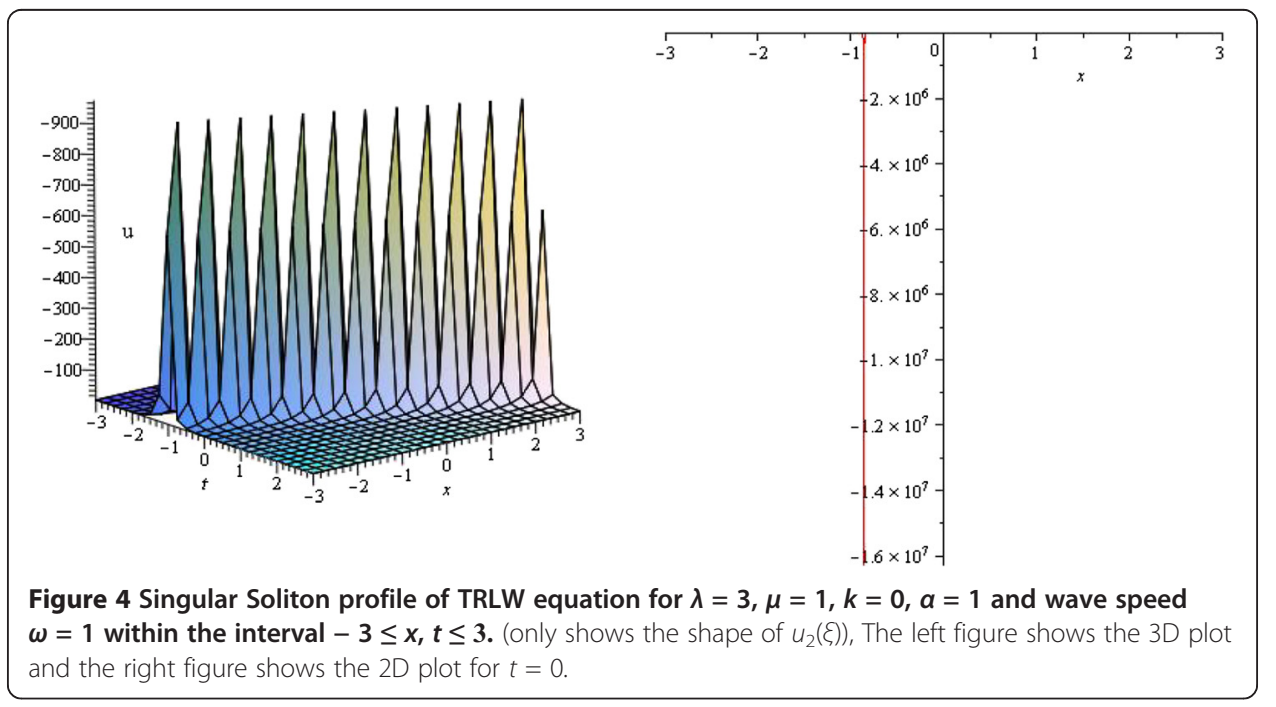




\section{Conclusions}

In this paper, we have utilized the $\exp (-\Phi(\xi))$-expansion method to seek exact solutions of the TRLW equation and KdV equation, and found new solutions. The performance of the $\exp (-\Phi(\xi))$-expansion method is reliable and effective. It can be concluded that this method is very powerful and efficient technique to find the exact solutions for a large class of problems and can be easily extended to all kinds of non linear evolution equations in mathematical physics and engineering.

Competing interests

The authors declare that they have no competing interests.

\section{Authors' contributions}

This work was carried out in collaboration among the authors. All authors have a good contribution to design the study, and to perform the analysis of this research work. All authors read and approved the final manuscript.

\section{Author details}

${ }^{1}$ Department of Mathematics, Pabna University of Science \& Technology, Pabna 6600, Bangladesh. ${ }^{2}$ Department of Applied Mathematics, University of Rajshahi, Rajshahi 6205, Bangladesh.

Received: 14 December 2014 Accepted: 17 February 2015

Published online: 12 March 2015

\section{References}

Abdou MA (2007) The extended tanh-method and its applications for solving nonlinear physical models. Appl Math Comput 190:988-996

Bekir A (2008) Application of the $\left(G^{\prime} / G\right)$ - expansion method for nonlinear evolution equations. Phys Lett A 372:3400-3406 Bona JL, Chen H (1999) Comparison of model equations for small-amplitude long waves. Nonl Anal 38:625-647 He JH, Wu XH (2006) Exp-function method for nonlinear wave equations. Chaos Solitons Fractals 30:700-708 Hereman W (2011) Shallow Water Waves and Solitary Waves. Mathematics of Complexity and Dynamical Systems., pp 1520-1532, doi:10.1007/978-1-4614-1806-1_96

Hereman W, Banerjee PP, Korpel A, Assanto G, van Immerzeele A, Meerpole A (1986) Exact solitary wave solutions of nonlinear evolution and wave equations using a direct algebraic method. J Phys A Math Gen 19(2):607-628

Islam ME, Khan K, Akbar MA, Islam R (2013) Traveling Wave Solutions Of Nonlinear Evolution Equation Via Enhanced (G'/G)-Expansion Method. GANIT J Bangladesh Math Soc 33:83-92, doi.org/10.3329/ganit.v33i0.17662

Jeffrey A (1978) Nonlinear wave propagation. Z Ang Math Mech (ZAMM) 58:T38-T56

Joseph RI, Egri R (1977) Another possible model equation for long waves in nonlinear dispersive systems. Phys Lett A 61:429-432

Khan K, Akbar MA (2014a) The exp(-Ф(६))-expansion method for finding Traveling Wave Solutions of Vakhnenko-Parkes Equation. Int J Dyn Syst Differential Equat 5(1):72-83

Khan K, and Akbar MA (2014b) Exact Solutions of the (2+1)-dimensional cubic Klein-Gordon Equation and the (3+1)-dimensional Zakharov-Kuznetsov Equation Using the Modified Simple Equation Method. J Asso Arab Uni Basic Appl Sci 15:74-81, (doi.org/10.1016/j.jaubas.2013.05.001)

Khan K, and Akbar MA (2014C) Traveling Wave Solutions of the (2+1)-dimensional Zoomeron Equation and Burgers Equation via the MSE Method and the Exp-function Method. Ain Shams Eng J 5:247-256m, (doi.org/10.1016/j. asej.2013.07.007)

Khan K, Akbar MA (2014d) Traveling Wave Solutions of Nonlinear Evolution Equations via the Enhanced (G'/G)-expansion Method. J Egypt Math Soc 22(2):220-226, doi:10.1016/j.joems.2013.07.009

Marchant TR, Smyth NF (1996) Soliton interaction for the extended Korteweg-de Vries equation. IMA J Appl Math 56:157-176

Monro S, Parkes EJ (1999) The derivation of a modified Zakharov-Kuznetsov equation and the stability of its solutions. J Plasma Phys 62(3):305-317

Monro S, Parkes EJ (2000) Stability of solitary-wave solutions to a modified Zakharov-Kuznetsov equation. J Plasma Phys 64(3):411-426

Parkes EJ, Duffy BR (1996) Travelling solitary wave solutions to a compound KdV Burgers equation. Cmput Phys Commun 98:288

Taghizade N, Neirameh A (2010) The solution of TRLW and Gardner Equations by the (G'/G)-Expansion Method. Int J Nonlinear Sci 9(3):305-310

Taghizadeha N, Mirzazadeha M, Paghaleh AS (2012) Exact travelling wave solutions of Joseph-Egri(TRLW) equation by the extended homogeneous balance method. Int J Appl Math Comput 4(1):96-104

Wang ML, Li XZ (2005a) Applications of Expansion to periodic wave solutions for a new Hamiltonian amplitude equation. Chaos, Solitons Fractals 24:1257-1268

Wang ML, Li X (2005b) Extended F-expansion and periodic wave solutions for the generalized Zakharov equations. Phys Lett A 343:48-54

Wang Z, Zhang HQ (2007) A new generalized Riccati equation rational expansion method to a class of nonlinear evolution equation with nonlinear terms of any order. Appl Math Comput 186:693-704

Wang M, Zhou Y (2003) The periodic wave equations for the Klein-Gordon-Schrodinger equations. Phys Lett A 318:84-92

Wang DS, Ren YJ, Zhang HQ (2005) Further extended sinh-cosh and sin-cos methods and new non traveling wave solutions of the (2+1)-dimensional dispersive long wave equations. Appl Math E Notes 5:157-163 
Wang ML, Li XZ, Zhang JL (2007) Sub-ODE method and solitary wave solutions for higher order nonlinear Schrodinger equation. Phys Lett A 363:96-101

Wang M, Li X, Zhang J (2008) The (G'/G)-expansion method and travelling wave solutions of non linear evolutions equations in mathematical physics. Phys Lett A 372:417-423

Wazwaz AM (2009) Partial Differential Equations and Solitary Waves Theory. Higher Education Press Beijing and Springer-Verlag, Berlin Heidelberg

Yan ZY (2001) New explicit travelling wave solutions for two new integrable coupled nonlinear evolution equations. Phys Lett A 292:100-106

Zakharov VE, Faddeev LP (1971) The Korteweg-de Vries equation: a completely integrable Hamiltonian system. Funct Anal Appl 5:280-287

Zayed EME, Gepreel KA, Horbaty MM (2008) Exact solutions for some nonlinear differential equations using complex hyperbolic function. Appl Anal 87:509-522

Zhang S, Tong JL, Wang W (2008) A generalized (G'/G)-expansion Method for the mKdV equation with variable coefficients. Phys Lett A 372:2254-2257

Submit your manuscript to a SpringerOpen ${ }^{\circ}$ journal and benefit from:

- Convenient online submission

- Rigorous peer review

- Immediate publication on acceptance

- Open access: articles freely available online

High visibility within the field

Retaining the copyright to your article

Submit your next manuscript at $>$ springeropen.com 